\title{
Pobreza, equidad y educación. Reto para Colombia como país miembro de la OCDE 2012-2017
}

\author{
Linda Cristiam Rincón Vargas
}

Camilo Andrés Cruz Suárez

Anderson Molina Sarria

Escuela Militar de Cadetes "General José María Córdova”

\section{RESUMEN}

Se analizan los desafíos de las políticas públicas de Juan Manuel Santos en el Plan Nacional de Desarrollo de Colombia, 2014-2017, para su ingreso a la Organización de Cooperación para el Desarrollo Económico. Se esboza la conceptualización y caracterización del desarrollo económico contemporáneo frente a la OCDE.

¿Por qué esta organización se mantiene vigente?, ¿cuáles son los objetivos frente a los países miembros y el resto del mundo? Estas preguntas que analizan bajo la perspectiva de la teoría de la interdependencia, se identifican los retos del Gobierno colombiano para ingresar a la organización y garantizar su sostenimiento, además, se analizan aspectos del desarrollo sostenible.

PALABRAS CLAVE

OCDE, conflicto, procesos de paz, interdependencia, desarrollo sostenible, educación, empleo, economía, desarrollo territorial.

\section{CITACIÓN}

Rincón, L.C., Cruz, C.A., Molina, A. (2019). Pobreza, equidad y educación, reto para Colombia como país miembro de la OCDE 2012-2017. Brújula, Semilleros de Investigación, 7 (13), 7-22. https://doi.org/10.21830/23460628.83 


\section{Introducción}

La OCDE es una organización que es reconocida por compartir políticas públicas que tienen como objetivo mejorar la gobernanza y la calidad de vida de los habitantes, como es considerado en su primer artículo en el Tratado de París del 30 de septiembre de 1961, por lo cual, recientemente, Colombia, tras iniciar el proceso de negociaciones para la paz en noviembre del año 2012, decide optar por la posibilidad de ingresar en pro de un desarrollo económico.

Pero ¿qué es la OCDE y por qué Colombia decidió ser parte de esa organización?, y, más allá de eso, ¿cuáles son los retos que en materia de equidad, pobreza y educación enfrenta Colombia para alcanzar un desarrollo económico sostenible desde su ingreso como miembro de la OCDE?

Es importante mencionar los objetivos de desarrollo sostenible, también denominados Objetivos Globales, liderados por las Naciones Unidas que, a este respecto, hace un llamado a la comunidad mundial para afrontar los grandes desafíos que enfrenta la humanidad, con el fin de permitir que todas las personas tengan igualdad de oportunidades y en busca de minimizar el impacto negativo al planeta.

Los 17 objetivos de desarrollo sostenible son: fin de la pobreza; hambre cero; salud y bienestar; educación de calidad; igualdad de género; agua limpia y saneamiento; energía asequible y no contaminante; trabajo decente y crecimiento económico; industria, innovación e infraestructura; reducción de las desigualdades; ciudades y comunidades sostenibles; producción y consumo responsables; acción por el clima; vida submarina; vida de ecosistemas terrestres; paz, justicia e instituciones sólidas y alianzas para lograr los objetivos propuestos.

\section{Metodología}

La presente investigación es cualitativa-descriptiva, ya que "proporciona profundidad a los datos, dispersión, riqueza interpretativa, contextualización del ambiente o entorno, detalles y experiencias únicas. También aporta un punto de vista fresco, natural y holístico de los fenómenos, asi como la flexibilidad” (Hernández, Fernández y Baptista, 2010). Una de las características de la investigación cualitativa es la orientación hacia la exploración, la descripción y el entendimiento.

\section{Creación y evolución del desarrollo económico sostenible y su materialización a través de la OCDE}

La cooperación internacional es un término que se utiliza mucho actualmente, de la misma manera que el de Relaciones Internacionales, esto obedece a un hecho que muestra la interdependencia de los Estados para coexistir, que en un principio tomó forma con el Tratado de Versalles, que se realizó casi inmediatamente después del fin de la Primera Guerra Mundial, que tuvo lugar desde 1914 hasta 1918.

La Gran Guerra había causado unos veintidós millones de muertos y lo que más deseaba Woodrow Wilson era que algo así no se repitiera jamás. De modo que el 8 de enero de 1918, pronuncia en el Congreso de su país un discurso en el que se incluía una lista de 14 puntos con el objetivo de la obtención de la paz, puntos que llegaron a ser el punto de partida para el Tratado de Versalles de 1919. El proyecto consistía en la creación de una organización supranacional de colaboración entre los países firmantes que habían salido victoriosos del conflicto reciente y cuto fin sería la consecución definitiva de la paz mundial: la Sociedad de Naciones, (López, 2016)

Sin embargo, el panorama internacional no permitió establecer una cooperación que trascen- 
diera en el tiempo, pues en vez de conseguir una sociedad internacional que cooperara de forma sostenible, los diferentes líderes aumentaron su poder, generado por la percepción de poner en riesgo la seguridad a nivel internacional. Para Robert O Keohane estos hechos se denominan “discordia”, lo que condujo a muchos Estados a perseguir, con todos sus recursos, la obtención y el cuidado de sus intereses, al final esto trajo como resultado la Segunda Guerra Mundial, que tuvo lugar de 1939 a 1945.

La Primera Guerra Mundial, que duró cuatro años, tres meses y catorce días, dejó profundos cambios en el territorio europeo. Las potencias vencedoras permitieron que se incumplieran ciertos términos establecidos en el Tratado de Versalles, lo que generó el resurgimiento del militarismo y un nacionalismo agresivo en Alemania, así como desórdenes sociales en varios países europeos.
Este hecho reformó a toda Europa como escenario donde la violencia tuvo furor y trascendió a todo el mundo, pues, tras finalizar la discordia internacional, se inicia el desarrollo económico contemporáneo y la cooperación internacional, que ha sido promovida por organizaciones que se han mantenido desde entonces.

Así las cosas, ¿qué espera el mundo después de una guerra más? La Primera Guerra Mundial no resolvió los conflictos, al contrario, los agudizó, y veinte años después ocasionó la Segunda Guerra Mundial. Sin embargo, bajo la voluntad de la presidencia de Woodrow Wilson se llegó a un cambio y una seguridad “obligada” se impondría:

Tras el conflicto, en el siglo XX se establecen varios interrogantes: ¿cómo, después de una destrucción masiva en Europa, iniciada por Hitler, podrían llegar décadas de paz y prosperidad en Europa? dicho escenario fue posible por tres razones:

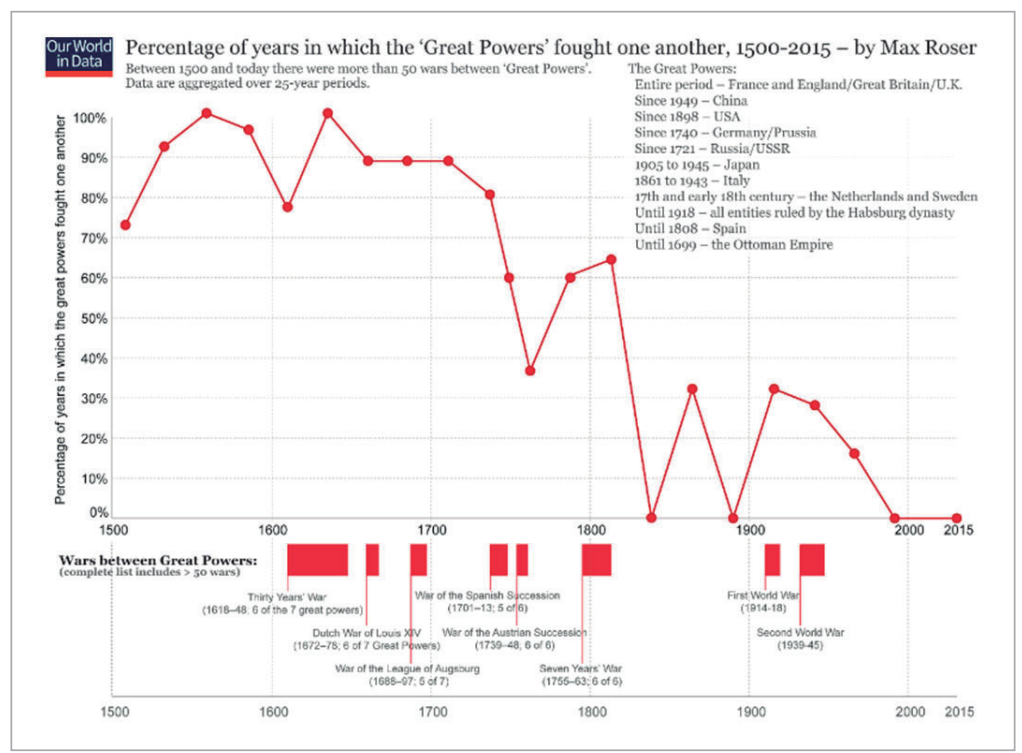

Figura 1. Los mejores ángeles de nuestra naturaleza: ¿por qué la violencia ha disminuido?, con base en información de Levy y Pinker.

Fuente: Recuperado de https://commons.wikimedia.org/wiki/File:Ourworldindata_percentage-of-years-in-whichthe-great-powers-fought-one-another-1500\%E2\%80\%932000.png 
1. La fatiga de la guerra

2. La devastada Alemania que surgió

3. La existencia de la amenaza nuclear (Kershaw, 2015)

Europa aparece como el continente que sirvió como iniciador para el desarrollo económico contemporáneo que hoy se conoce. Fue el plan de reconstrucción de Europa que, a su vez, dio inicio a la OEEC "Organization for European Economic Co-operation” (Organización para la Cooperación Europea). Organización que apoyó al continente que había sido víctima de la guerra, el mismo que tras varias ayudas se conocería, a partir de 1961, como la OCDE u oecd, por sus siglas en inglés. Por otra parte, es el inicio de varias organizaciones como la Organización de Naciones Unidas (ONU) y otros organismos supranacionales que fueron creados durante la hegemonía de los Estados Unidos, que se posicionaba como un líder mundial.

Los diferentes organismos y regímenes que han existido se crearon en hegemonía, pero por muchos sucesos Estados Unidos deja de tener esta hegemonía, su poder protagónico entra en declive a partir de 1960, e incluso hoy es un hecho bastante evidente ya que no es el único actor con peso económico. Pero, ¿por qué las organizaciones creadas en su régimen se mantienen? Esto es explicado por Robert O. Keohane así:

Para aquellos que creen en la teoría de estabilidad hegemónica en cuestiones de relaciones político-económicas la cooperación es imposible de obtener ya que los intereses transgreden e interrumpirán los de los otros, lo que finalmente guía a los Estados a una fragmentación política sin un Estado superior que coordine sus esfuerzos y sus garantías, lo que permite orientarse a un Estado poderoso que guíe y perdure en el tiempo, pero son difíciles de ser creadas y es por ello que las organizaciones que fueron creadas en la reconstrucción de Europa se mantienen y a su vez son reformadas. (Keohane, 1984)
Teniendo en cuenta lo anterior, el desarrollo económico contemporáneo se describe, en muchas de las organizaciones, como la forma en que los Estados luchan contra la pobreza, lo que introduce el término "desarrollo sostenible", que se refiere a cómo la humanidad evoluciona positivamente a través del tiempo conservando el medioambiente, donde este último subsiste como se plantea en el primer tratado que formalizó la OCDE:

Ya que el artículo 1 firmado en París el 30 de septiembre de 1961, afirma que la organización de cooperación y desarrollo económico promoverá las políticas de triunfar en el máximo desarrollo económico y empleo mejorando la calidad de vida de sus habitantes, promoviendo también el desarrollo económico de los países pertenecientes a la organización como los que no hacen parte de él y contribuyendo en aumentar los tratados internacionales sin ningún tipo de discriminación” (OCDE, 1997).

Como también lo hace la organización más importante que es conocida como la ONU, que manifiesta:

La Carta de Naciones Unidas fue, y sigue siendo, un enérgico llamado a mantener la paz y la seguridad internacionales, y a promover el desarrollo económico y social. Los fundadores se inspiraron en la traumática experiencia de dos guerras mundiales, el sufrimiento de la humanidad y un profundo deseo de paz duradera basada en la igualdad, la dignidad y el progreso social y económico. (Harston, 2015, p. 24)

Por último, la lucha contra la pobreza y en pro del desarrollo sostenible hacen parte del desarrollo económico contemporáneo, esta es la perspectiva con la cual muchas organizaciones, incluso la OCDE, ayudan a diferentes Estados a mejorar sus políticas públicas para poder apoyar a los demás, es esa la perspectiva que condujo a Colombia a querer hacer parte de tal organización. 


\section{El proceso de ingreso de Colombia a la OCDE}

La Organización para la Cooperación y el Desarrollo Económico (OCDE) es una organización internacional de carácter intergubernamental; fue fundada el 30 de septiembre de 1961 y tiene su sede en París. En la actualidad cuenta con 37 países, es un grupo destacado por tener políticas públicas que promueven un desarrollo considerable a nivel interno. Los países miembros tienen por objetivo dos aspectos fundamentales: "la prosperidad económica [...] esencial para la preservación de las libertades individuales y el mejoramiento del bienestar general y la cooperación entre pares es una herramienta fundamental para alcanzarla” (Velásquez, 2014).

Para el ingreso de un país, este debe demostrar a la organización que tiene la capacidad de cumplir con los objetivos que la OCDE se ha propuesto, desde 1960, para mejorar el desarrollo económico, estos son:

- Económicos

- Sociales

- Medioambientales

- Buen gobierno

- Los retos que trae la globalización

Estos aspectos son inherentes a la misión que posee de lograr la expansión económica y de empleo a nivel mundial.

\section{Indicadores de pobreza, equidad y educación}

Para ingresar a la OCDE Colombia debe luchar contra la pobreza a través del desarrollo sostenible, esto se puede lograr mediante la implementación de políticas públicas que aumenten las capacidades estatales, para ello Juan Manuel Santos estableció su política de gobierno “paz, equidad y educación”, destinada a cumplirse por medio del Plan Nacional de Desarrollo.

El ingreso de Colombia a la OCDE implica un trabajo complejo ante las dificultades de pobreza e inequidad latentes que generan condiciones difíciles, e incluso conflictos, que representan un obstáculo para el desarrollo sostenible de la población colombiana:

En primer lugar, el PNUD (Programa de Naciones unidas para el Desarrollo), que actualmente acoge 177 países y territorios ayudando a los gobernantes y sus habitantes para superar desafíos que tienen en comunidad, define la pobreza no solo como la falta de alimentos o dinero que tenga una persona, sino como la falta de capacidades del ser humano para ser y hacer, como, por ejemplo, la posibilidad de estudiar, transportarse, acceder a mercados y demás. La medida que se tiene para Colombia comprende tan solo 3 indicadores, estos son:

- Necesidades básicas insatisfechas.

- Índice de pobreza multidimensional.

- Pobreza monetaria.

\section{Necesidades básicas insatisfechas}

El índice de necesidades básicas insatisfechas es una herramienta que se utiliza para saber cuáles hogares se encuentran en condiciones precarias o simplemente no llegan a los estándares necesarios para satisfacer las necesidades de todos los integrantes de la familia. Este índice tiene varios criterios, como el consumo, el acceso a la educación, una vida digna y otros criterios que evalúa para medir la calidad de vida de una región en específico. Para ello sigue varios procesos, el primero se llama "identificación”, que consiste en la realización de una encuesta sobre los bienes y 
servicios disponibles; el segundo proceso mide los recursos del hogar, sus ingresos y gastos, para saber si resulta suficiente para una vida aceptable (Feres y Mancero, 2001).

\section{Índice de pobreza multidimensional}

Es una medida que complementa las bases de ingreso y se centra en el individuo y diferentes aspectos necesarios para llevar una vida normal, las cuales se muestran en la figura 2. Este índice fue creado por la Oxford Poverty \& Human Development Initiative (OPHI), junto con el programa de Desarrollo de la UN (United Nations Development Programme).

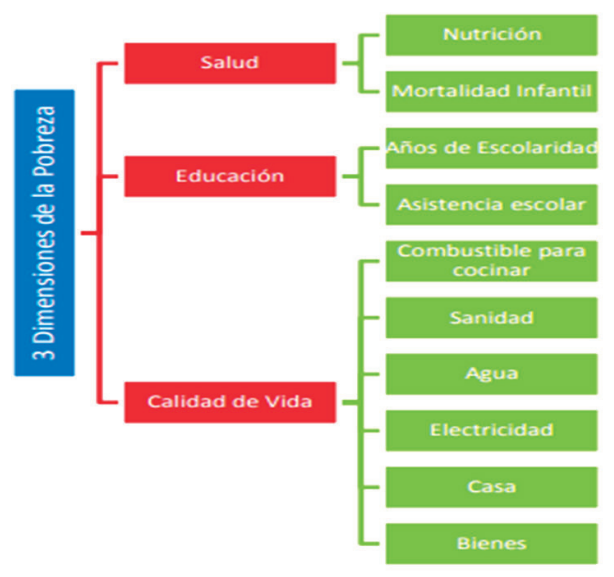

Figura 2. La pobreza

Fuente: Oxford \& Human Development Initiative. Recuperado de http:/ www.ophi.org.uk/multidimensional-poverty-index

\section{Pobreza monetaria}

Es un índice que mide la pobreza de la siguiente manera:

se considera como pobres monetarios a las personas que residen en hogares cuyo gasto per cápita es insuficiente para adquirir una canasta básica de alimentos y no alimentos (vivienda, vestido, educación, salud, transporte, etc.). Son pobres extremos aque- llas personas que integran hogares cuyos gastos per cápita están por debajo del costo de la canasta básica de alimentos. (inei, 2017)

Dejando el tema de los índices de pobreza a un lado, se observa que el reto de un país para luchar por reducir la pobreza es algo bastante exigente, ya que elevar los estándares de vida depende de aumentar las riquezas por medio del comercio y de otras herramientas que deben ser ofrecidas por el Estado. Con base en lo anterior, Colombia es un país que puede otorgar todas estas capacidades económicas, incluso la revisión que hizo la OCDE recientemente solo muestra resultados satisfactorios frente a los recursos de Colombia:

Colombia es el quinto país más grande en extensión y el tercero de mayor población de América Latina, con una superficie de 1,1 millones de $\mathrm{km}^{2}$ y una población de 47 millones de personas. Es el único país de América del Sur que tiene salida directa al Océano Atlántico y al Pacífico. Cuenta con abundantes recursos agrícolas y de agua dulce, una biodiversidad excepcional y una gran riqueza en recursos naturales como el níquel, cobre, hierro, carbón, gas natural, petróleo, oro, plata, platino y esmeraldas (oecd, 2015).

Leer este tipo de anexos de estudios internacionales es algo impresionante, Colombia no es cualquier país, pero saber que Colombia lleva más de 50 años de conflicto hace a muchos teóricos preguntarse el porqué de ello. En este estudio no se encuentra la hipótesis de que el odio bipartidista, que inició el 9 de Abril de 1948, se ha mantenido hasta la fecha durante más de 60 años y es la posible respuesta al interrogante de los teóricos, en cambio, se propone que la respuesta está en la desigualdad social del país, lo demás es añadidura a lo inevitable.

En el mapa los colores más oscuros representan los sectores donde las Necesidades Básicas Insatisfechas (NBI) afectan más, lo que es impresionante es que la situación para esos años 


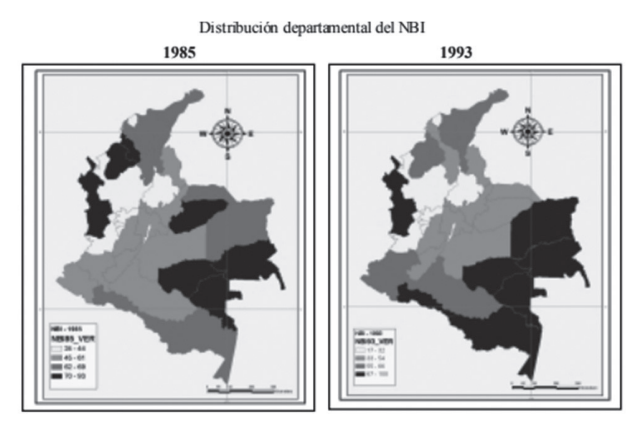

Figura 3. Economía regional, Dimensión espacial de la pobreza.

Fuente: Recuperado de http://www.banrep.gov.co/ sites/default/files/publicaciones/archivos/DTSER54.pdf

no mejoraba y en esos territorios el conflicto interno parecía incrementar, lo que hace preguntar a muchas personas en varios lugares del mundo: ¿qué está pasando con los recursos de una tierra tan fructífera?

La existencia de la pobreza en una sociedad depende del nivel de ganancias y de su distribución, mentir sobre estos dos factores generaría un gran impacto en el capital humano, en función del mercado, el desempleo y la distribución de los bienes y servicios por parte del Gobierno.
Pero si se analizan las ganancias de Colombia, la situación es perfecta para que nadie sea pobre, pero la verdad es que la inequidad pinta otro escenario, ya que el $45 \%$ de las ganancias las obtienen los ricos, el 10\% la población común y tan solo el 14\% lo reciben los dueños de tierras, lo que sucede, al menos desde 1860, en Cundinamarca (Robinson, 2016).

Las condiciones internas fueron el principal componente que inició el conflicto armado en Colombia, que existe desde 1948, cuando Jorge Eliecer Gaitán fue asesinado:

El asesinato de Gaitán produce un estallido en Bogotá y otras ciudades, que no derroca al gobierno, pero desencadena la violencia campesina en dos "márgenes cercanos": en los llanos orientales, donde la guerrilla es más disciplinadamente liberal, en zonas de minifundio andino $\mathrm{y}$, en particular, cafetero. (PNUD, 2003)

Pero hablar del conflicto, la violencia directa de las guerrillas contra la fuerza pública y la lucha por el control del narcotráfico en Colombia sería limitarse a impresiones de la gente del común. No son fundamentales para entender la poca capacidad de Colombia para generar un

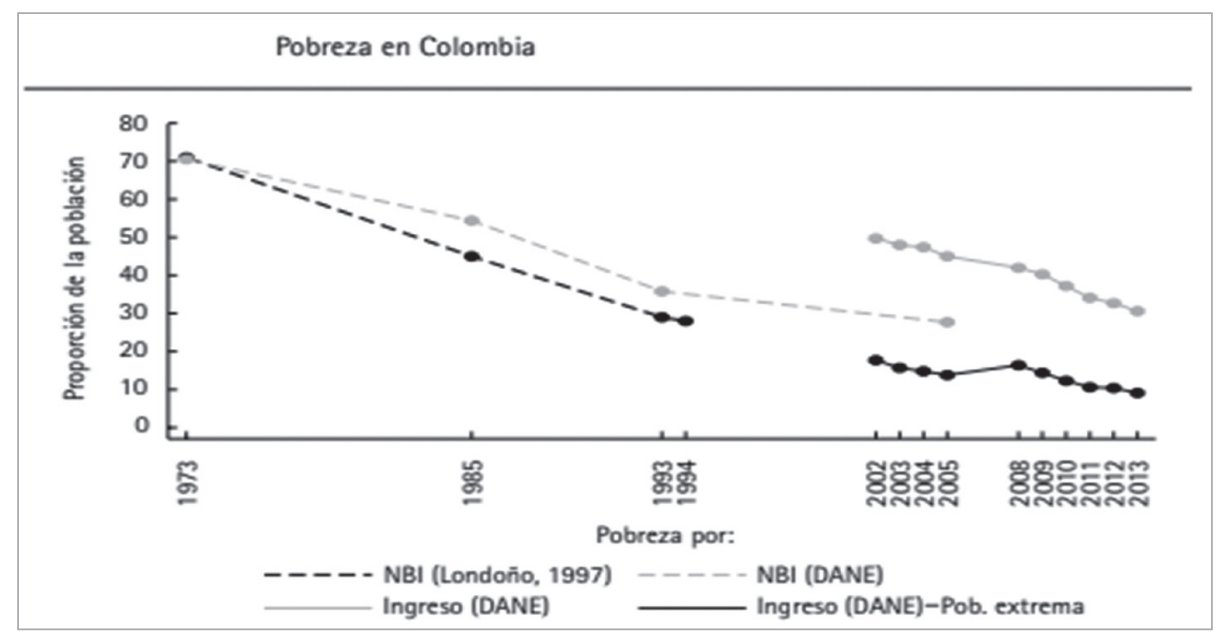

Figura 4. La miseria en Colombia

Fuente: Robinson (2016). 
desarrollo sostenible, como lo menciona el doctor César Augusto Niño González, magíster en Seguridad y Defensa Nacional:

El conflicto armado en Colombia tiene sus raíces desde los 1940`s pero se oficializa en 1960 siendo la génesis que guiaría el país a un conflicto que no termina hasta el día de hoy, dio como resultado dos grupos guerrilleros los cuales son las farc y el eln que señalaron el futuro conflictivo de Colombia por la tenencia territorial consecuencia del olvido estatal, hecho del cual gran parte de la culpa se le atribuye al Estado por generar condiciones fatales al desconectarse de zonas cruciales donde la delincuencia, las muertes y la desigualdad tomarían lugar. Además de señalar enemigos a actores internos que no compartían ideas con los del partido conservador. (Niño, 2017, p. 327)

De tal manera que el simple cese de las hostilidades no traerá el desarrollo sostenible que necesita el país. Lo que en muchos sectores se conoce como "paz", donde el conflicto terminaría y jamás se volvería a presentar, termina siendo un simple acontecimiento que se resume en dejar de luchar por algún tiempo y retomar cuando las soluciones de la desigualdad social estén presentes, lo que permite la germinación de esa semilla de amenaza conocida como disidencia. Mientras no sea el Estado el que garantice mejor vida a sus ciudadanos, ninguna parte va a estar satisfecha, como se explica en la teoría de conflictos de Johan Galtung, pues

los conflictos no son algo negativo o positivo solo son un proceso natural que simplemente ocurren en las sociedades, muchas de ellas ocurren por diferencias ideológicas o culturales, pero por medio de un método se puede sacar lo mejor del conflicto, para que las partes queden satisfechas y la sociedad en sí evolucione. De no ser así y lo que resulte siendo una paz falsa las partes jamás quedaran satisfechas y solo será cuestión de tiempo para que el conflicto y demás fenómenos sociales continúen. (Calderón, 2009)

En adición a lo anteriormente mencionado, es preciso afirmar que el proceso de diálogos de paz que tuvo lugar en La Habana, Cuba, durante los últimos años, no ha sido el único proceso de paz existente, han sido varios los intentos fallidos por construir una paz estable, pero lamentablemente no se logra una consolidación efectiva para los actores involucrados. A continuación, se abordarán los principales procesos de paz fallidos y sus características.

En primer lugar es necesario establecer que el gobierno nacional no solo ha entablado negociaciones de paz con las guerrillas, incluso la primera negociación - conocida como el Frente Nacional— que tuvo lugar desde 1954 hasta 1978, fue una regulación para que los partidos tradicionales, conservador y liberal, tuvieran las mismas oportunidades de participación en el gobierno nacional, incluso uno de los procesos de paz del cual se va a hablar se desarrolló con paramilitares. En muchas ocasiones, se observa que los líderes de cada bando parecen estar dispuestos a cumplir con lo pactado, pero lastimosamente el conflicto muta y continúa incluso con quienes estaban dispuestos a acatar lo negociado:

Las negociaciones que ha tenido Colombia con las farc no son la primera ni la segunda negociación sino que ya son varias veces que el gobierno nacional se ha sentado a negociar, en total han sido 7 los presidentes que han llegado a este escenario: Belisario Betancur, Virgilio Barco, Gaviria, Ernesto Samper, Pastrana, Álvaro Uribe y la última negociación con el expresidente Juan Manuel Santos (Villarraga, 2015).

El Frente Nacional fue el primer tratado fallido, se dio en la época en que surgieron las guerrillas modernas que hoy se conocen, comprendida entre 1958 y 1974:

La idea del Frente Nacional (FN) se originó en la estrategia de colaboración de los dos partidos tradicionales en el manejo del poder político durante la administración de Olaya, pero fue López Pumarejo 
quien en 1946, cuando ya la violencia se salía de madre, propuso que los liberales presentaran a los conservadores una lista de presidenciables para que estos escogieran, El gran opositor de la colaboración entre los partidos tradicionales había sido Lauriano Gómez, pero en 1957, tras el exilio en España, aceptó la iniciativa liberal de turnarse el poder de los dos partidos. Para los liberales y muchos conservadores -así no lo confesaran-, la violencia tenía que ver con el botín burocrático. La creencia de que la coalición liquidaría la lucha banderiza no resultó cierta. El vacío fue llenado por el MRL y la Anapo, movimientos que en última instancia trasladaron su fuerza social a los grupos armados. No en vano el MRL tuvo que ver con la fundación del ELN y la Anapo con el M-19 (Molano, 2016).

El siguiente tratado de paz fue un intento de Belisario Betancourt, cuyas conversaciones se iniciaron en 1982 y terminaron en 1986, que más que acabar con la violencia directa iría por algo más allá que dejar la lucha de las partes por el poder:

El proceso de paz de Betancur fue pionero y aportó elementos que aparecen ahora en las políticas de paz de otros gobiernos”, asegura Álvaro Villarraga, historiador y exintegrante del Centro de Memoria Histórica. Se resalta de su mandato el haber reconocido a las organizaciones insurgentes como actores políticos, hizo una importante convocatoria nacional por la paz, logró negociaciones y acuerdos por primera vez en la historia reciente, dirigidos al cese de las hostilidades y la solución de la guerra.

El expresidente introdujo el reconocimiento de que había que tratar y superar "las causas objetivas y subjetivas” del conflicto y que debe haber razones, ante todo políticas, para reconocer tanto el alzamiento en armas como su solución.

Belisario Betancur fue un empecinado con la solución negociada al conflicto y para lograrlo se enfrentó a lo más fuerte de la clase política. Como él mismo lo reconoció, eso fue lo que más le costó: "Hay compatriotas que aún no están dispuestos a sacrificar sus privilegios, aunque sean causa de zozobra para el país entero” (Forero, 2018)

El tercer intento, tras más años de conflicto, llegaría de la mano del entonces presidente César Augusto Gaviria Trujillo, quien llegó a ser secretario general de la Organización de Estados Americanos, en cuyo gobierno tuvo lugar la Asamblea Nacional Constituyente de la cual surge la Constitución Política de 1991:

Este proceso de paz dejó como resultado la desmovilización de varios grupos armados como son:

- El Ejército de Liberación Popular.

- Partido Revolucionario de los Trabajadores.

- El movimiento armado Quintín Lame.

- La corriente de renovación socialista.

Pero a pesar de todos estos esfuerzos quedaron los grupos que se mantendrían hasta el año 2012, los cuales son las farc y el eln, razón por la cual el conflicto simplemente cambia de personas que laboran, pero jamás se detuvo. (Villarraga, 2015)

Por último, y con no muy buenos resultados, se hizo un intento en el gobierno de Andrés Pastrana, abogado, empresario, diplomático, periodista y político colombiano, miembro y líder del Partido Conservador Colombiano, donde las partes acordaron reunirse e incluso hubo un cese de fuego bilateral que se hizo solo para darle tiempo de fortalecerse a las partes para seguir combatiendo, específicamente en el gobierno de Álvaro Uribe Vélez, lo que dejó al país desconcertado y sin guía para llegar a la paz que ya bastante habían negociado.

Por esta razón, una negociación más no representa mucho para la población civil, incluso si se habla de los diálogos en La Habana, Cuba. Es correcto decir que las personas no esperan nada positivo, incluso si el conflicto acabara solo piensan en la posibilidad de más conflicto.

Santos ha evitado los errores cometidos por ex presidente Andrés Pastrana (1998 - 2002) en negociaciones con la guerrilla de las FARC. A diferencia de Pastrana, Santos no anunció su intención de negociar durante su elección presi- 


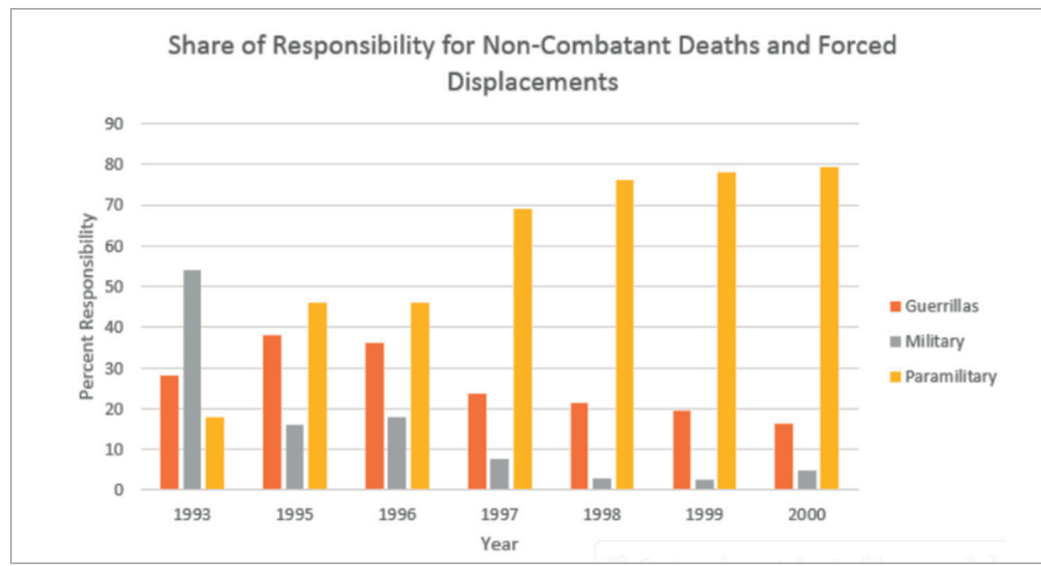

Figura 5. Una breve historia del imperialismo estadounidense y la violencia estatal en Colombia Fuente: Recuperado de http:// kurukshetra1.wordpress.com/2015/09/27/a-brief-history-of-imperialism-and-stateviolence-in-colombia

dencial Campaña. Después de asumir el cargo, Inicialmente se involucró en secreto pre-negociaciones. Estas conversaciones preliminares resultaron en una estricta agenda limitada de seis temas - desarrollo agrícola, participación política, terminando el conflicto, drogas ilegales, víctimas del conflicto e implementación del tratado. (Nussio, 2016)

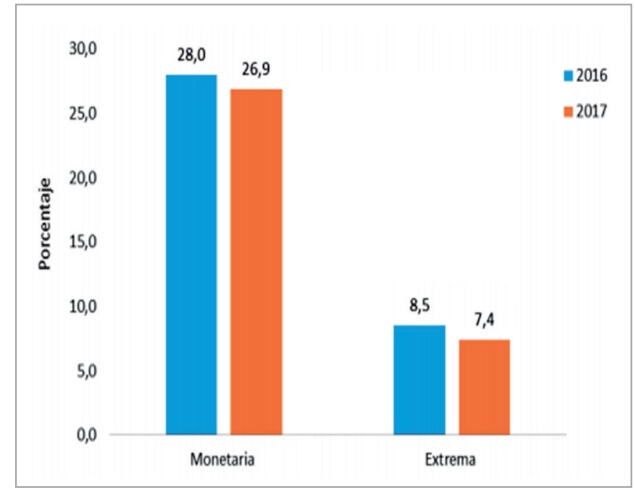

Figura 6. Incidencia de la pobreza monetaria y de la pobreza extrema total nacional, 2016-2017.

Fuente: dane y geih.

Pero aparte de la cantidad de procesos de paz, hay una razón más fundamental por la cual la población tiene una mala percepción frente a estas didácticas, ya que al final terminarán haciendo de las suyas, solo que, con otro logo, otro lema y los mismos males, es por eso por lo que tras estas dinámicas la población espera lo mismo que ha sucedido durante ya varios años.

En la década de los 90' se experimentó una reducción del tamaño del Estado en América Latina, debido al aumento de las privatizaciones de empresas públicas; cambios en las funciones estatales, al perder el Estado el papel de productor de bienes y servicios; creciente descentralización de los servicios y también de los recursos; aparición de nuevos actores en las políticas públicas no estatales, donde los organismos no gubernamentales adquieren presencia en la entrega de servicios públicos y promueven la participación y el control comunitarios. En ese marco, también cambiaron los enfoques orientados a superar la pobreza, lo que se tradujo en más recursos, nuevas instituciones y programas, modalidades de gestión de las políticas y programas más descentralizados y participativos y con mayor injerencia de la sociedad civil, y la aplicación de nuevos instrumentos de asignación de recursos. En síntesis, hacia la década del 2000, junto con un aumento en los niveles de pobreza de la población, aparecen nuevas formas de gestión pública que reorientan los esfuerzos estatales hacia programas encaminados a disminuir la pobreza, en especial la extrema pobreza. (Rincón, 2014) 


\section{El Estado colombiano desde los indicadores estudiados para alcanzar un desarrollo económico sostenible en el marco de la OCDE}

Resulta imperioso hacer un llamado a conservar la seguridad multidimensional en Colombia, sin embargo, para alcanzar esta meta hay que tener en cuenta que dentro del Estado se presentan aspectos problemáticos como los que se enuncian a continuación:

- Proliferación del porte de armas de forma ilegal.

- Terrorismo.

- Delincuencia organizada.

- Ciberseguridad.

- Seguridad energética.

- Cambio climático.

Por lo anterior, la preocupación por el desarrollo económico atañe también a la preocupación de las fuerzas militares en cuanto que ninguno de los aspectos mencionados llegue a decaer $y$, por consiguiente, a generar ambientes donde las amenazas puedan surgir, como ha ocurrido en Colombia desde hace ya varios años.

De tal manera que es primordial garantizar a los ciudadanos el buen servicio de su Estado, en respuesta a ello, en el año 2012, acontecen dos grandes hechos, uno de ellos es la iniciación de los diálogos de paz y el otro es hacer público el interés de formar parte de diferentes organizaciones internacionales, como lo son la otan y la OCDE. Por consiguiente, se plantea el Plan Nacional de Desarrollo -2014 2017, bajo el lema "paz, equidad y educación” como respuesta en cuanto a políticas públicas para que Colombia responda al reto que exige pertenecer a tan destacadas organizaciones. El Plan Nacional de Desarrollo se reglamentó por medio de la ley 1753 de 2015 que entró en vigencia el martes 9 de junio. En su Artículo primero reza:

Objetivos del Plan Nacional de Desarrollo. El Plan Nacional de Desarrollo 2014-2017 “Todos por un nuevo país”, que se expide por medio de la presente ley, tiene como objetivo construir una Colombia en paz, equitativa y educada, en armonía con los propósitos del Gobierno Nacional, con las mejores prácticas y estándares internacionales, y con la visión de planificación, de largo plazo prevista por los objetivos de desarrollo sostenible. (Congreso de la República, 2015)

Para analizar las políticas del plan de desarrollo es pertinente explicar cuáles son los estándares que plantea la OCDE. Para iniciar, el proceso de adición no es algo rápido, de hecho, es algo lento y muy beneficioso, ya que los participantes se reúnen cuando un país está a punto de iniciar su proceso de adición y juntos establecen una hoja de ruta, la de Colombia es la [C (2013)110/FINAL], donde se fijan los términos, las condiciones y procedimientos.

Como miembro de la OCDE lo que debe primar es lo escrito en el artículo número 1. Dice que debe promover políticas públicas tendientes a:

- Mantener el mayor índice de desarrollo sostenible y aumento del empleo.

- Promover la expansión económica a Estados que pertenecen y los que no.

- Ayudar a expandir los tratados internacionales.

Para lograr este paso tiene que pasar por diferentes Comités, se nombran algunos de ellos, como:

- Gobernanza pública

- Inversiones

- Medio ambiente

- Desarrollo territorial 
- Economía y desarrollo

- Educación

- Empleo

- Salud

- Tratados

Al pasar por estos Comités Colombia solo puede escoger entre: la aceptación, aceptación con un espacio de tiempo para su implementación, aceptación con observaciones y anulación. (oecd, 2013)

Por lo anteriormente expuesto, las políticas públicas que son de interés de estudio en el presente documento, tienen como eje principal la crítica al lema del Plan Nacional de Desarrollo "paz, equidad y educación". Se plantea el interrogante si el Estado puede efectivamente garantizar las condiciones, para que la población logre el disfrute de la paz. En caso de no contar con dicha garantía implica replantear la ecuación, así:

\section{"Educacion+equidad=paz"}

Es cierto que Colombia tiene muchísimas políticas por reformar que son mencionadas en el artículo 1753 del 2015, como infraestructura, creación de recibos electrónicos para mejorar el análisis de datos de inversiones económicas en el país e inversión en la ciencia de tecnología y comunicaciones, pero todas estas temáticas, a fin de cuentas, no van a surtir efecto, ya que el avance es poco y no es suficiente para llegar a los estándares que exige la OCDE. Por ello se analizarán solo dos aspectos que resultan fundamentales para mejorar las condiciones de vida de los habitantes de un país, estos son:

- Educación.

- Economía y desarrollo.

\section{Educación en el Plan Nacional de Desarrollo}

La educación el primer factor que podría aumentar la igualdad; el documento escrito por Robert Keohane en su apología a la interdependencia, "Cooperation and discord in the world political economy”, explica lo que es discordia, esta se ve reflejada cuando el Estado no limita la satisfacción de sus intereses y toma lo que la población necesita, al mencionar esto se hace

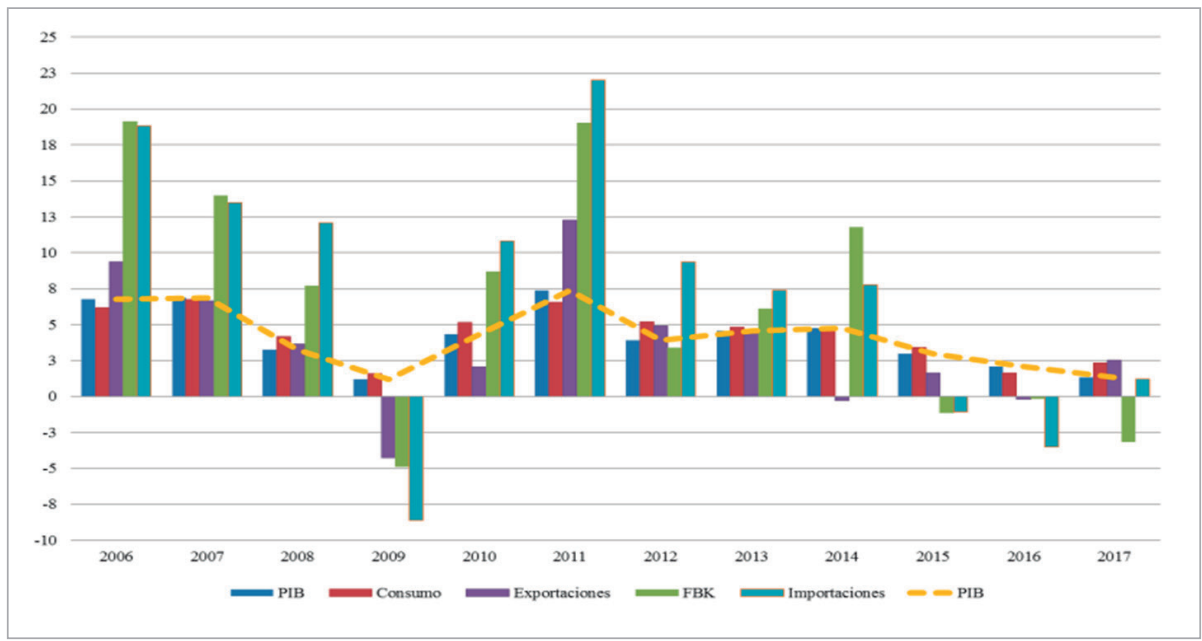

Figura 7. Producto Interno bruto (pib), (precios constantes de 2015). Variación porcentual anual. Fuente: Cuentas Nacionales, Departamento Administrativo Nacional de Estadística (dane). 


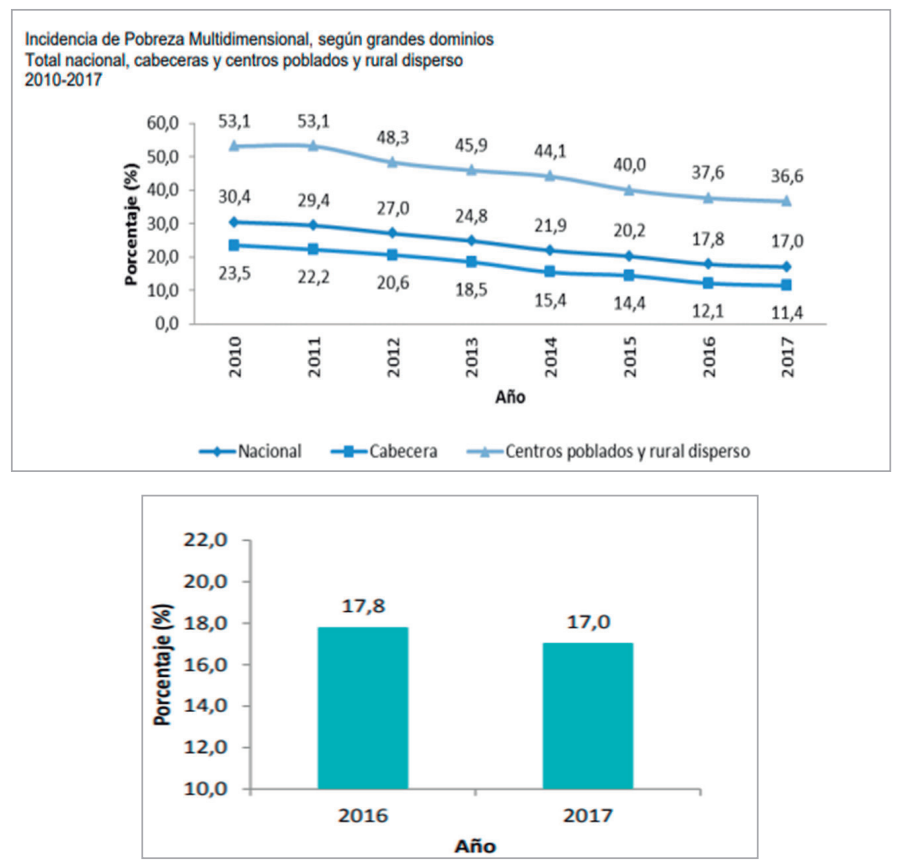

Figura 8. Boletín técnico pobreza monetaria y multidimensional en Colombia, 2017 (dane).

Fuente: Recuperado de https://www.dane.gov.co/files/investigaciones/condiciones_vida/pobreza/bol_pobreza_17.pdf

referencia a muchas situaciones que surgen de ello, dos de estas son la poca capacidad de gestión y los altos niveles de corrupción que se presentan en el Estado.
La deficiente capacidad y coordinación en los niveles de gestión es uno de los mayores retos estructurales que han contribuido a empeorar las inequidades regionales y territoriales. La corrupción sigue siendo un problema grave, alimentado por la burocracia ineficiente del gobierno y la falta de transparencia en la información. (oecd, 2016).

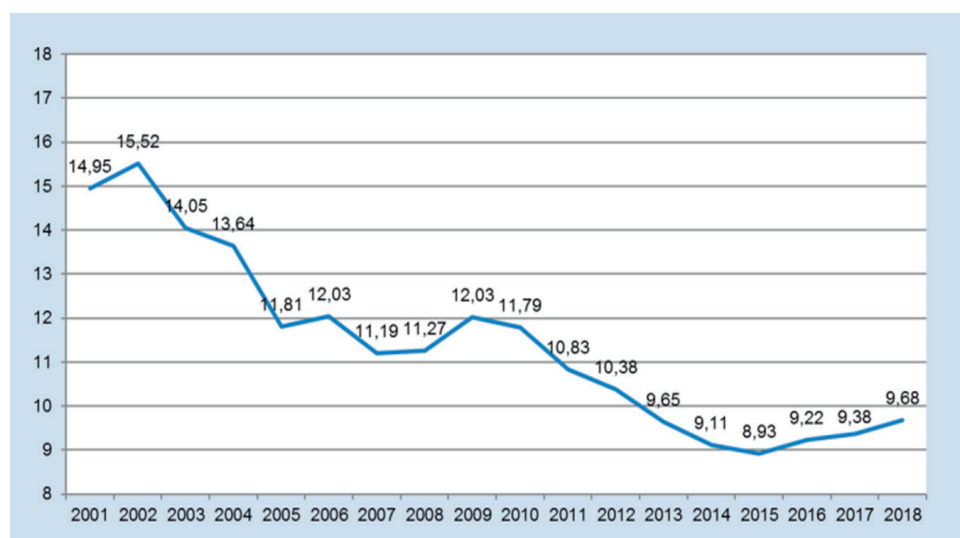

Figura 9. Tasa de desempleo en Colombia

Fuente: Encuesta Continua de Hogares, dane. 
Tras analizar esto, es posible afirmar que es la educación el factor en el que hace falta trabajar para acabar con las necesidades de la población, pues es el factor que ha determinado que las economías de muchos otros países latinoamericanos no hayan evolucionado.

Porque desde la colonia española los países latinoamericanos han jugado el papel de “proveedores de bienes primarios o básicos” y su posibilidad de industrialización se ha visto opacada, lo que los ha limitado solo a la posibilidad de vender sus recursos a muy bajo precio y a depender de las exportaciones hasta la actualidad.
Un producto que venda Colombia cambia su precio dependiendo de varios factores, uno de ellos es la capacidad de vender a través de diferentes medios y otro es su calidad, de hecho, la venta de papa, café y diferentes productos se dificulta, ya que son anticuados. A pesar de ello, Colombia ofrece buena calidad para su exportación, ¿pero qué de los productos que se venden en el interior del país?, es allí donde el estudio de diferentes aspectos se hace necesario para mejorar la productividad en el campo, más allá de los cultivos ilícitos que han sido exportados a nivel mundial.

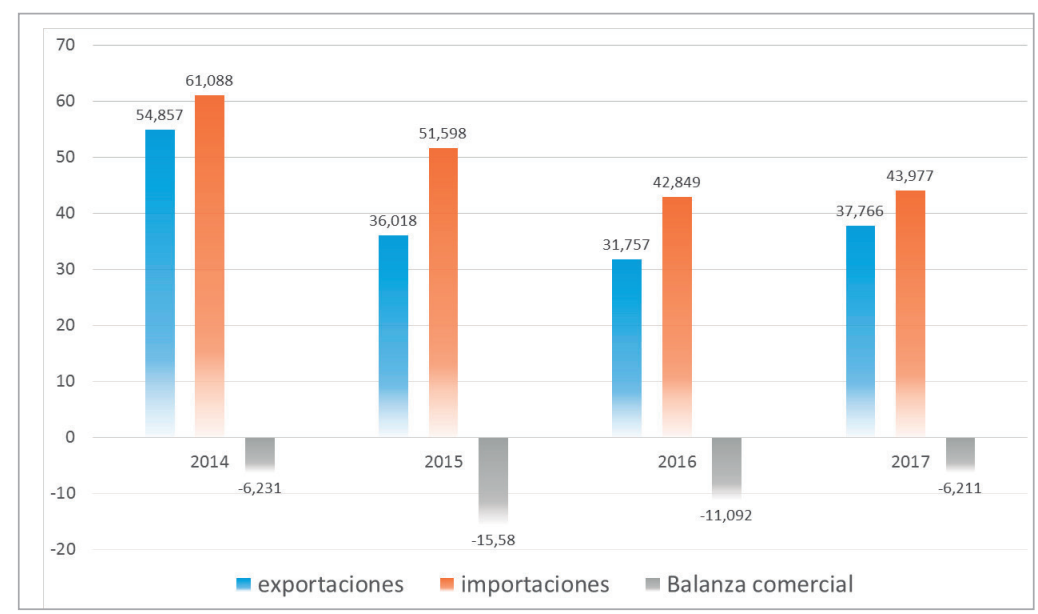

Figura 10. Balanza comercial de Colombia (anual en millones de dólares)

Fuente: Recuperado de http://repository.urosario.edu.co/bitstream/handle/10336/18739/MonroyCuello-Maria\%20 Alejandra-2018.pdf?sequence=1\&isAllowed=y

\begin{tabular}{|c|c|c|c|c|c|c|c|c|c|c|c|c|c|c|c|c|c|}
\hline & \multicolumn{3}{|c|}{$\begin{array}{l}\text { CSM } \\
\text { (netos) }\end{array}$} & \multicolumn{3}{|c|}{$\begin{array}{l}\text { Kalmanovitz } \\
\text { (netos) }\end{array}$} & \multicolumn{2}{|c|}{$\begin{array}{l}\text { Sarmiento } \\
\text { (brutos) }\end{array}$} & \multicolumn{3}{|c|}{$\begin{array}{l}\text { O'Byme y Reina } \\
\text { (netos) }\end{array}$} & \multicolumn{6}{|c|}{$\begin{array}{l}\text { Rocha } \\
\text { (netos) }\end{array}$} \\
\hline & marih. & coca & total & marih. & coca & total & $\begin{array}{l}\text { coca } \\
\text { min. }\end{array}$ & $\begin{array}{l}\text { coca } \\
\max .\end{array}$ & coca & $\begin{array}{l}\text { latex } \\
\text { heroina }\end{array}$ & total & marih. & $\begin{array}{l}\text { coca } \\
\text { min. }\end{array}$ & $\begin{array}{l}\text { coca } \\
\text { max. }\end{array}$ & heroi. & $\begin{array}{l}\text { total } \\
\text { min. }\end{array}$ & $\begin{array}{l}\text { total } \\
\text { max. }\end{array}$ \\
\hline $76-80$ & & & & 480 & 1848 & 2328 & & & & & & & & & & & \\
\hline $81-82$ & 188 & 2846 & 3033 & 250 & 3840 & 4090 & 2031 & 4188 & & & & & & & & & \\
\hline $83.86^{\circ}$ & 87 & 1528 & 1614 & 121 & 4080 & 4201 & 1496 & 3723 & 2602 & & 2602 & 66 & 939 & 3579 & & 1005 & 3644 \\
\hline 87.91 & 51 & 684 & 735 & 173 & 3869 & 4042 & & & 2903 & 196 & 3099 & 39 & 473 & 4624 & 5 & 517 & 4668 \\
\hline 92.94 & & & & & & & & & & & & 125 & 294 & 2982 & 286 & 705 & 3393 \\
\hline
\end{tabular}

Figura 11. Los ingresos de Colombia producto de la exportación de drogas ilícitas Recuperado de https://www.repository.fedesarrollo.org.co/handle/11445/2193 


\section{Economía y desarrollo}

El desarrollo económico se genera por muchos factores, el más importante de ellos es la evolución social que requiere para que este ocurra.

El desarrollo económico se define como el proceso en virtud del cual la renta real per cápita de un país aumenta durante un largo periodo de tiempo. En otros términos, el desarrollo es un proceso integral, socioeconómico, que implica la expansión continua del potencial económico, el auto sostenimiento de esa expansión en el mejoramiento total de la sociedad. (Castillo, 2011, p. 2).

\section{Conclusiones}

Colombia ha tenido un avance muy lento pero progresivo. Buscar la negociación para alcanzar la paz o combatir las diferentes amenazas, como las guerrillas y el narcotráfico, no son caminos que conduzcan a la tan anhelada evolución que las evite, por otro lado, con la globalización las amenazas emergentes evolucionan en maneras que hacen muy difícil su identificación.

Probablemente el ingreso a la OCDE sea el inicio para mejorar la calidad de vida en el país, pero también da pie a muchas preguntas que hay para responder sobre el desarrollo económico contemporáneo de Colombia: ¿es válido llegar a integrarse en cuestión de políticas públicas a las élites mundiales?, ¿Colombia ha encontrado la clave para terminar con el Conflicto armado?.

Lo que al final queda claro, es que Colombia aceptó el reto, su ruta de acceso ya se planteó y se aceptó su ingreso, es cuestión de que las políticas públicas en materia de paz, equidad y educación sean el eje principal del país para los años venideros, pero ¿podrán los nuevos gobernantes pensar en estos ejes para adoptar sus políticas públicas y llegar a tan anhelado desarrollo económico sostenible, para así reemplazar el crimen y el narcotráfico con nuevos productos y oportunidades para las personas que viven constantemente un país apunto de surgir?

\section{Declaración de divulgación}

Los puntos de vista y los resultados de este artículo pertenecen a los autores y no reflejan necesariamente los de la Escuela Militar de Cadetes “General José María Córdova”.

\section{Financiamiento}

Los autores no declaran fuente de financiamiento para la realización de este artículo.

\section{Sobre los autores}

Linda Cristiam Rincón Vargas. Magister en Ciencia Política, Universidad de Salerno - Italia y Universidad Católica de Colombia. Docente Facultad de Relaciones Internacionales, Escuela Militar de Cadetes "General José María Córdova”. Contacto: linda.rincon@esmic.edu.co

Camilo Andrés Cruz Suárez. Semillero de investigación CITIUS de la Facultad de Relaciones Internacionales, estudiante VIII semestre, Escuela Militar de Cadetes "General José María Córdova”.

Anderson Molina Sarria. Semillero de investigación CITIUS de la Facultad de Relaciones Internacionales, estudiante VIII semestre, Escuela Militar de Cadetes “General José María Córdova”.

\section{Referencias}

Castillo Martín, P. (2011). Política económica: crecimiento económico, desarrollo económico, desarrollo sostenible. Revista internacional del mundo económico y del derecho, 3(1), 1-2.

Concha, P. C. (2009). Teoría de conflictos de Johan Galtung. Revista de paz y conflictos, (2), 60-81.

Congreso de la República (2015). Ley 1753 de 2015. Diario Oficial 49538, 1-2. 
Feres, J. C. y Mancero, X. (2001). El método de las necesidades básicas insatisfechas (nbi) y sus aplicaciones en América Latina. Cepal.

Forero, Javier. La paz de Belisario: una esperanza presa de la confrontación política. Revista Semana. Recuperado de https://www.eltiempo.com/ politica/gobierno/procesos-de-paz-que-lidero-belisario-betancur-expresidente-de-colombia-302436

Harston, J. (2015). Introduction to the un System: Orientation for Serving on a un Field Mission. Peace Operatoin Trainig Institute, 20-35.

inei (2017). Informe Técnico: Evolución de la Pobreza Monetaria 2007-2016. Recuperado de https://www.inei.gob.pe/media/cifras_de_ pobreza/pobreza2016.pdf

Keohane, R. O. (1984). After Hegemony: Cooperation and Discord in the World Political Economy Princeton. Press, Princeton.

Kershaw, I. Out of the ashes: Europe's rebirth after the Second World War, 1945-1949. Journal of the British Academy, 3, 167-183.

López, E. (2016). Etiquetas. Ética y política, democracia y Naciones Unidas, Paz, RMS Lusitania, Sociedad de Naciones, Thomas Woodrow Wilson. Recuperado desde: https://estebanlopezgonzalez. com/2016/12/13/thomas-woodrow-wilson/

Melo, O. (2018). Modelos de gobernabilidad migratoria: entre la integración regional y la Securitización, una aproximación a la diáspora venezolana. En IV ciclo de conferencias "Implicaciones regionales de la situación actual en Venezuela". Ponencia llevada a cabo en la Escuela Militar de Cadetes "General José María Córdova", Bogotá.

Molano, A. (2016). Fragmentos de la historia del conflicto armado (1920-2010). Contribución al entendimiento del conflicto armado en Colombia. Comisión histórica del Conflicto y sus víctimas.
Ediciones desde abajo. Segunda reimpresión. 596-597.

Niño, C. A. (2017). Breve historia del conflicto armado en Colombia. Revista de Paz y Conflictos, 0(1), 327-330.

Nussio, E. (2016). Peace and Violence in Colombia. CSS Analyses in Security Policy. Recuperado de https://css.ethz.ch/content/dam/ethz/special-interest/gess/cis/center-for-securities-studies/pdfs/ CSSAnalyse-191-EN.pdf., 2.

oecd (1997). Explorations in oeec history. Francia: oecd series, 1-2.

oecd (2013). C 110/FINAL Unclassified. English/ French Council, 1-3.

oecd (2015). Review of Agricultural Policies: Colombia 2015, 6 .

oecd (2016). Education in Colombia, Reviews of National Policies for Education, oecd Publishing, Paris. doi.org/10.1787/9789264250604-en

PNUD (2003). El conflicto, callejón con salida, informe nacional de desarrollo humano para Colombia. Recuperado de http://hdr.undp.org/ sites/default/files/colombia_2003_sp.pdf

Rincón L. C. (2014). Desigualdad económica y social en Colombia: las políticas públicas para la reducción de la pobreza (Tesis de maestría en Ciencia Política).Universidad Católica de Colombia, Universidad de Salerno Italia.

Robinson, J. A. (2016). La miseria en Colombia. Revista Desarrollo y Sociedad, (76), 9-88. doi:10.13043/dys.76.1

Stone, W. (1992). A Brief of History. Washington D. C.: Center of Military History.

Velásquez, D. (2014). OCDE, ingreso e implicaciones para Colombia. Recuperado de https://repository.upb.edu.co/bitstream/ handle/20.500.11912/2873/Art\%C3\%ADculo\%20de\%20grado-Diana\%20C.\%20 Vel\%C3\%A1squez\%20M.pdf?sequence=1\&isAllowed $=\mathrm{y}$ 